\title{
A Fundamental Limitation for Bipolar Transistor Scaling
}

\author{
Y. PAN
}

\begin{abstract}
A microscopic model of minority-carrier diffusion in a heavily doped emitter is proposed. Monte Carlo simulation demonstrates that statistical fluctuation in the base current is one of the fundamental limitations in high-speed applications of scaled bipolar transistors.
\end{abstract}

\section{INTRODUCTION}

TN RECENT YEARS, the down-scaling of bipolar transisItors has led to a very significant decrease in the switching delay of circuits and is reaching into the sub-50-ps range of the ECL gate [1]. Previous theories [1] on the limitations of bipolar transistor scaling emphasize mainly phenomena like the shallow-base punchthrough, power dissipation, and emitter contact resistance when a poly-emitter is utilized.

The shrinkage of both lateral and vertical dimensions requires more heavily doped emitters and bases to reduce their resistance. This leads to a significant reduction in minority-carrier numbers in these regions. A simple calculation using the updated experimental data of bandgap narrowing [2] suggests that a uniformly doped emitter with a volume of $1.0 \mu \mathrm{m}^{3}$ and a donor density of $10^{20} \mathrm{~cm}^{-3}$ will include no more than 60 holes under $0.7-\mathrm{V}$ emitter/base bias at $300 \mathrm{~K}$. For such a small "ensemble," statistical fluctuation of minority-carrier transport should be carefully investigated.

Classical methods [3] for the calculation of diffusion current noise are complex and are usually based on small-signal approaches. The validity is questionable when large fluctuation phenomena are studied. In contrast, it has been pointed out by Moglestue [4] that the Monte Carlo method is well suited for noise studies as it reflects the basic transport physics. In his approach, however, the fluctuation was exaggerated due to the usage of "super particles." In a heavily doped emitter, the minority-carrier ensemble is small. Therefore, it is easy to follow the entire time history of all the carriers. This makes the Monte Carlo method a powerful tool to investigate the fluctuation of minority-carrier diffusion in heavily doped emitters.

\section{MODELS}

A "simplest" n-p-n transistor with a uniform doping profile will be studied here. A metallic contact on the emitter serves as an ideal sink for the holes. The donor concentration

Manuscript received June 18, 1990

The author is with the Department of Electrical Engineering, Electrical Materials Laboratory, Delft University of Technology, Mekelweg 4, 2600 GA, Delft, The Netherlands. IEEE Log Number 9038772 in the emitter is $10^{20} \mathrm{~cm}^{-3}$. The typical emitter junction depth is $0.1 \mu \mathrm{m}$ and emitter area is $10 \mu \mathrm{m}^{2}$. For such an emitter area, the sidewall injection is not important. Therefore, a one-dimensional approach is adopted. ShockleyRead-Hall and Auger recombination in the emitter will be neglected. Because an electric field is not present in the neutral emitter region, diffusion is the only form of hole transport. We also neglect recombination current in the base region and other nonideal components for the base current. Thus the hole current at the emitter/base boundary is equal to the base current and the fluctuation of this component will be reflected in the common-emitter current gain.

Holes injected from the base are in thermal equilibrium with each other and a Maxwellian velocity distribution can be used. The mean-free-path for holes is $\lambda=D_{p} / v_{t h}$ [5] with $D_{p}$ as the hole diffusion coefficient and $v_{t h}$ as the "one-sided mean velocity" as introduced by Persky [6]. $\lambda$ is about 100 $\AA$ at $300 \mathrm{~K}$ when the experimental data for the diffusion coefficient of [2] are utilzied. The emitter then is divided into ten subregions with a width of $\lambda$. The holes in each subregion can be emitted to successive subregions with a random velocity $v(i)$. The electrical current across the boundary of subregion $i-1$ and $i$ is

$$
I_{i-1, i}=\frac{Q_{i-1, i}}{\delta t}
$$

where $\delta t$ is the measurement time and $Q_{i-1, i}$ is the net charge flow across the boundary during the measurement time. If the excess hole number in each subregion is $P_{i}$, we have

$$
I_{i-1, i}=\frac{q \int_{t}^{t+\delta t} d t\left\{\sum_{i=1}^{P_{i}} \tau^{-1}(i)-\sum_{i=1}^{P_{i-1}} \tau^{-1}(i)\right\}}{\delta t}
$$

where $\tau(i)=\lambda / v(i)$ is the one-sided random free flight time.

\section{Simulation}

Initially an emitter/base bias $V_{b e}$ is applied to the emitter/base junction. Holes will then be injected to the emitter due to the lowered emitter/base barrier. We suppose that the number of holes in the first subregion responds to the $V_{b e}$ instantaneously and is fixed to

$$
P_{1}=A_{e} \lambda p_{0} \exp \frac{q V_{b e}}{k T}
$$

where $A_{e}$ is the emitter area and $p_{0}$ is the equilibrium hole 


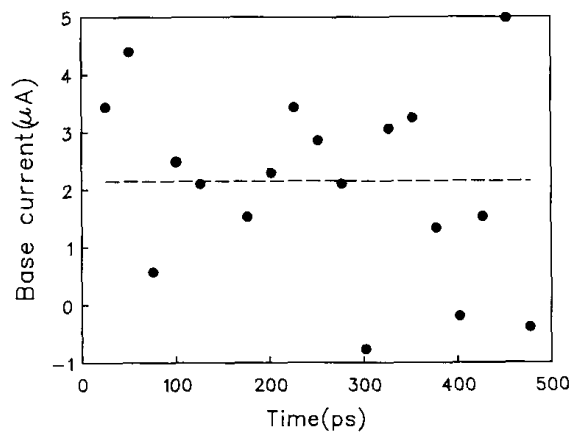

Fig. 1. Base current versus time. $A_{e}=10 \mu \mathrm{m}^{2}, \delta t=5 \tau_{\text {ave }}=0.85 \mathrm{ps}$, $W_{e}=0.1 \mu \mathrm{m}$, and $V_{b e}=0.75 \mathrm{~V}$.

concentration. As time proceeds, holes will move towards the emitter contact. At a specific time, a hole in the subregion $i$ can be emitted thermionically to either the right or the left subregion, if the free flight time is less than the mean free flight time, which is taken as the time step in our simulation. The mean free flight time is

$$
\tau_{\text {ave }}=\frac{1}{2} \cdot \frac{\lambda}{v_{t h}} .
$$

The prefactor $\frac{1}{2}$ accounts for two possible directions for the hole emission. $\tau_{\text {ave }}$ is about $1.7 \times 10^{-13} \mathrm{~s}$ in our simulation. According to the Maxwellian velocity distribution, the free flight time for a specific hole at a specific time is

$$
t_{f}(i)=\frac{1}{2} \cdot \frac{\lambda}{\left(\frac{k T}{m^{*}}\right)^{1 / 2}\left|\Gamma_{1}\right|}
$$

where $\Gamma_{1}$ is a random number with Gaussian-distributed deviates with zero mean and unit variance. The direction of the hole jumping is completely random and determined by a Boole-type random number $\Gamma_{2}$. After the hole jumps to another subregion, the time step will be updated as the difference between the previous time step and the free flight time. The hole will continue to move until the generated free flight time is longer than the updated time step. The hole transport is controlled by the random numbers $\Gamma_{1}$ and $\Gamma_{2}$. The holes in the last subregion may be absorbed by the emitter contact. The simulation will continue until the specific end time is reached. The position of the holes will be recorded as a function of time.

The calculated base current as a function of time is depicted in Fig. 1 with an emitter area of $10.0 \mu \mathrm{m}^{2}$, a measurement time of $5 \tau_{\text {ave }}$, and an emitter/base bias of $0.75 \mathrm{~V}$. The dashed line in the figure is based on the classical expression for the base current [7]. The fluctuation is indeed significant. The relative deviation of the base current as a function of measurement time $\delta t$ is depicted in Fig. 2 for three different emitter areas. The relative deviation is defined as the ratio of standard deviation to mean value and is determined over 100 data points. We find that increasing measurement time or emitter area results in reduction of the fluctuation. The junction depth dependence of the deviation is also illustrated

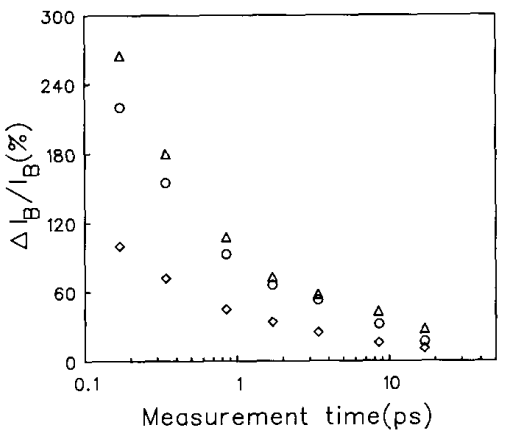

Fig. 2. Relative deviation of the base current versus measurement time. $W_{e}=0.1 \mu \mathrm{m}$ and $V_{b e}=0.75 \mathrm{~V} . \triangle A_{e}=5.0 \mu \mathrm{m}^{2}, \bigcirc A_{e}=10.0$ $\mu \mathrm{m}^{2}$, and $\diamond A_{e}=50.0 \mu \mathrm{m}^{2}$.

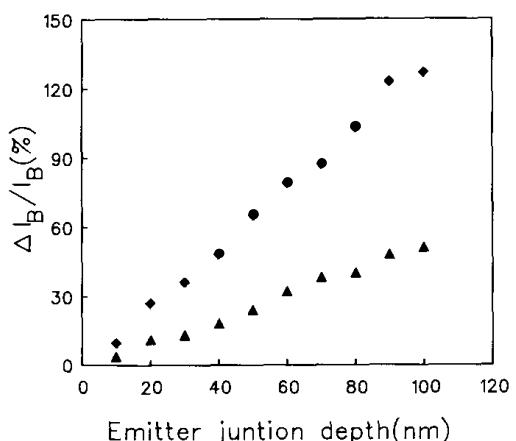

Fig. 3. Relative deviation of the base current versus emitter junction depth. $A_{e}=10.0 \mu \mathrm{m}^{2}$, and $\delta t=10 \tau_{\text {ave }}=1.7 \mathrm{ps} . \forall V_{b e}=0.7 \mathrm{~V}, \Delta V_{b e}=$ $0.75 \mathrm{~V}$.

in Fig. 3 for two different biases. Decreasing the junction depth or increasing the bias increases the base current and so decreases the fluctuation.

\section{ConClusion}

The above results imply that when bipolar transistors are scaled to smaller dimensions, the fluctuation in base current, and consequently the common-emitter current gain is quite significant. For the transistor investigated in this letter with $5.0-\mu \mathrm{m}^{2}$ emitter area, $0.1-\mu \mathrm{m}$ junction depth, 8.5-ps measurement time, and $0.75-\mathrm{V}$ emitter/base bias, the base current deviation is $43 \%$. This sets up the maximum operating 
frequency for the transistor. More lightly doped emitters (such as for heterojunction bipolar transistors) will relax this limitation but at a cost of increased contact resistance, especially when poly-emitters are utilized. Increasing the emitter/base bias will also make the base current more deterministic, but the other limitations like power dissipation and contact resistance will become more obvious. We conclude that the base current fluctuation is a fundamental limitation in bipolar transistor scaling.

\section{ACKNOWLEDGMENT}

The author wishes to thank Prof. M. Kleefstra and Dr. W. Crans for stimulating discussions and critically reading the manuscript.

\section{REFERENCES}

[1] D. Tang, "Scaling the silicon bipolar transistor," in Submicron Integrated Circuits, R. K. Watts, Ed. New York: Wiley, 1989

[2] J. del Alamo and R. M. Swanson, "Measurement of steady-state minority-carrier transport parameters in heavily doped n-type silicon," IEEE Trans. Electron Devices, vol. ED-32, p. 455, 1987.

[3] K. M. van Vliet, A. Friedmann, R. J. J. Zijlstra, A. Gisolf, and A. van der Ziel, "Noise in single injection diodes. I. A survey of methods," J. Appl. Phys., vol. 46, p. 1804, 1975.

[4] C. Moglestue, "A Monte Carlo particle study of the intrinsic noise figure in GaAs MESFET's," IEEE Trans. Electron Devices, vol. ED-32, p. 2092, 1985.

[5] R. S. Muller and T. I. Kamins, Device Electronics for Integrated Circuits. New York: Wiley, 1977, p. 30.

[6] G. Persky, "Thermionic saturation of diffusion current in transistors," Solid State Electron, vol. 15, p. 1345, 1972.

[7] M. Kleefstra, "A simple method of calculating the minority carrier current in heavily doped silicon," Solid-State Electron., vol. 28, pp. 991-995, 1985 